\title{
A Note on Advantages of the Fuzzy Gabor Filter in Object and Text Detection
}

\author{
Vladimir Tadic ${ }^{1, *}$, Tatjana Loncar-Turukalo ${ }^{2}{ }^{\circledR}$, Akos Odry $^{1}{ }^{1}$, Zeljen Trpovski ${ }^{2}$, Attila Toth ${ }^{3}$, Zoltan Vizvari ${ }^{4}$ \\ and Peter Odry ${ }^{1}$
}

check for updates

Citation: Tadic, V.; Loncar-Turukalo, T.; Odry, A.; Trpovski, Z.; Toth, A.; Vizvari, Z.; Odry, P. A Note on Advantages of the Fuzzy Gabor Filter in Object and Text Detection. Symmetry 2021, 13, 678. https:// doi.org/10.3390/sym13040678

Academic Editor: Dumitru Baleanu

Received: 2 March 2021

Accepted: 12 April 2021

Published: 14 April 2021

Publisher's Note: MDPI stays neutral with regard to jurisdictional claims in published maps and institutional affiliations.

Copyright: (C) 2021 by the authors. Licensee MDPI, Basel, Switzerland. This article is an open access article distributed under the terms and conditions of the Creative Commons Attribution (CC BY) license (https:/ / creativecommons.org/licenses/by/ $4.0 /)$.
1 Institute of Information Technology, University of Dunaujvaros, Tancsics Mihaly u. 1/A Pf.: 152, H-2401 Dunaujvaros, Hungary; odrya@uniduna.hu (A.O.); podry@uniduna.hu (P.O.)

2 Faculty of Technical Sciences, University of Novi Sad, Trg Dositeja Obradovića 6, 21000 Novi Sad, Serbia; turukalo@uns.ac.rs (T.L.-T.); zeljen@uns.ac.rs (Z.T.)

3 Institute of Physiology, Medical School, University of Pecs, Szigeti Str. 12, H-7624 Pecs, Hungary; attila.toth@aok.pte.hu

4 Department of Environmental Engineering, Faculty of Engineering and Information Technology, University of Pecs, Boszorkany Str. 2, H-7624 Pecs, Hungary; vizvari.zoltan@mik.pte.hu

* Correspondence: tadityv@uniduna.hu or laslo.tadic@gmail.com

\begin{abstract}
This note presents a fuzzy optimization of Gabor filter-based object and text detection. The derivation of a 2D Gabor filter and the guidelines for the fuzzification of the filter parameters are described. The fuzzy Gabor filter proved to be a robust text an object detection method in low-quality input images as extensively evaluated in the problem of license plate localization. The extended set of examples confirmed that the fuzzy optimized Gabor filter with adequately fuzzified parameters detected the desired license plate texture components and highly improved the object detection when compared to the classic Gabor filter. The robustness of the proposed approach was further demonstrated on other images of various origin containing text and different textures, captured using low-cost or modest quality acquisition procedures. The possibility to fine tune the fuzzification procedure to better suit certain applications offers the potential to further boost detection performance.
\end{abstract}

Keywords: text detection; Gabor filter; filtering; fuzzy optimization; fuzzification; robust license plate detection

\section{Introduction}

Texture and edge detection are important for detecting objects in complex images as they usually contain the most relevant information of an image. The texture and edge detection procedures have been extensively analyzed in image processing and computer vision in recent decades. Grayscale images exhibit increased variance at edge positions and texture changes. Conventional edge and texture detection methods, such as Sobel or Canny detectors, often detect edges by the maximum of the first derivative or the zero crossing points of the second derivative. Unfortunately, they are unable to simulate the performance of the human visual system in edge detection, as it exploits both powerful physiological apparatus and psychological abilities [1-3], because they are very sensitive to noise, even to small fluctuations in the brightness of the pixels in the image.

The Gabor filter has an important advantage over these standard edge detectors, as it can simulate the receptive field of the visual cortex [1-5]. It has been extensively used in various image processing tasks, such as text localization and object detection, while the extent and breadth of applications report its value when complexity vs. performance is observed. Daugman [1] noted that the Gabor filter can achieve the best localization performance, both in the spatial and frequency domains, via analyzing the uncertainty of the signal in orientation and in spatial frequency. This means that the interesting features of the target can be obtained by changing parameters of the Gabor filter according to the 
specific visual needs. The literature evidence confirms an extensive use of Gabor filter for numerous computer vision and image processing tasks, such as edge, texture, and object detection [1-30]. Mehrotra [3] introduced a method for edge detection based on Gabor filters. He showed that the best result of one-dimensional (1D) step edge detection is achieved when the product of the frequency and spatial deviation of the odd Gabor filter is equal to 1. In addition, he noted that this conclusion was also applicable to a two-dimensional (2D) step edge. Liu et al. [5] explained the Gabor filter-based edge detector method using simple mathematical derivation in the frequency domain. They concluded that the frequency domain is more adequate for the analysis of edges than the spatial domain. Allagwail et al. [6] presented a face recognition algorithm where the Gabor filter was used for feature detection and extraction with the local binary pattern and gray level co-occurrence matrix. The introduced method was developed using the Olivetti Research Laboratory and the Yale database. The authors stated that the method had a $100 \%$ face recognition accuracy on the tested database. Yu et al. [7] developed a saliency guided visual tracking algorithm using a correlation filter and a log-Gabor filter. The goal of their research was to improve the robustness of the tracking under obstruction and boundary effect challenges. The Gabor filter was used in the preprocessing for texture detection. Their experiments on standard datasets demonstrated the improved discrimination and interpretability of the suggested tracker when compared with other tracking algorithms. Zhang et al. [8] proposed a new method using an adaptive Gabor filter that outperforms all state-of-the-art algorithms in finger-vein recognition. The authors merged the Gabor filter with a convolutional neural network to determinate the gradient of the Gabor filter parameters. Wu et al. [9] combined the Gabor filter with morphological operations in order to detect residential areas in historical topographic maps, achieving an excellent performance in positional accuracy and in integrity of the residential boundary. Jing et al. [10] introduced a Gabor filter-based algorithm to assess the direction navigability of a geomagnetic field. The Gabor filter was used in the preprocessing for detection of the direction suitable-matching features. The parameters of the Gabor filter were optimized using the hybrid particle swarm optimization. They showed that the parameters of the Gabor filter had a significant impact on the direction suitable-matching features. Low et al. [11] proposed a novel face recognition method based on a multi-fold Gabor filter. They condensed 40 multi-scale and multi-orientated Gabor filters into eight filters for face recognition. Capizzi et al. [12] proposed an algorithm for multidimensional finite impulse response (FIR) filter design based on a Gabor system generated with a Gaussian function. The authors demonstrated that the developed method provided a better pass-band flatness than other known filter design approaches. They noted that the obtained improved performance of the 2D filters was suitable for use in space applications. Luan et al. [13] introduced a novel type of convolutional neural network, the Gabor Convolutional Networks (GCN), which proved to be highly robust against orientation and scale alterations according to conducted experiments. Bai et al. [14] applied a Gabor filter for skeletonization in noisy text images. The new skeleton filter was composed of a pair of oppositely oriented Gabor filters. Medina et al. [15] proposed a method for detection of cracks in concrete tunnel surfaces based on rotation invariant Gabor filters and image sensors, where the filter parameters were tuned by a modified genetic algorithm based on a differential evolution optimization method. Using the proposed filter, the cracks could be detected in all directions with an accuracy of $95.27 \%$. Meng et al. [16] introduced an improved circular Gabor filter-based algorithm for retinal identification. The developed procedure was tested on public retina databases combining the rotation and scaling. The authors reported encouraging results and robustness to rotations and scale variations.

In recent years, a new approach using fuzzy logic [31,32] has been introduced in the implementation of the Gabor filter [17-21]. Tadic et al. [17-19] approached the problem of filtration using Gabor filters by taking into account fuzzy logic, a powerful mathematical apparatus that closely imitates human reasoning [20]. Using this apparatus, the Gabor filter parameters were converted into the fuzzy parameters to improve the filter's response $[20,21]$. The results of this new filtering method contribute to more robust detec- 
tion and extraction of the desired objects from complex images, i.e., license plates, text, various textures, and circular and near-circular objects.

The intention of this article is to highlight the most relevant aspects of our research on the topic of Gabor filter fuzzification during the past few years [17-19]. The paper revises derivation on a 2D Gabor filter with an explanation of the fuzzification of the filter parameters and a guidance for the development of other fuzzy image filters. We highlight the impact of the fuzzified filter in different applications, with a focus on the problem of reliable detection of license plates and text areas from images obtained with low-cost cameras. These applications are the relevant segment of security and surveillance systems and should successfully deliver despite the presence of noise in acquired images, lighting conditions, artifacts, and other image-deteriorating effects. The localization of a license plate from a complex image, as one of the very first applications of object recognition, still remains a common research issue, with focus shifting towards boosting of the algorithm's performance in less controlled ambient conditions using commercial equipment [17-19,33-35]. Simple, fast, and robust solutions, such as the proposed fuzzy Gabor filter, open the possibility of using inexpensive cameras for license plate and text localization and similar basic computer vision tasks.

This paper is organized as follows: Section 2 reflects on derivation of the fuzzified Gabor filter and provides guidance on the fuzzification process. Section 3 presents and discusses the results, and concluding remarks are provided in Section 4.

\section{Fuzzification of the Gabor Filters}

\subsection{Gabor Filter Formulation}

The complex Gabor function in the spatial domain is represented as [4-6,17-20]:

$$
g(x, y)=s(x, y) w_{r}(x, y)
$$

where the function $s(x, y)$ represents the complex sine wave, and the function $w_{r}(x, y)$ represents the 2D Gaussian.

The complex sine wave is defined as:

$$
s(x, y)=e^{j\left(2 \pi\left(u_{0} x+v_{0} y\right)+\varphi\right)}
$$

where the $\left(u_{0}, v_{0}\right)$ denotes spatial frequencies, and the $\varphi$ is the phase of the filter.

Parameters $\left(u_{0}, v_{0}\right)$ represent spatial frequencies in Cartesian coordinates. These spatial frequencies can be represented in polar coordinates as follows:

$$
\begin{aligned}
& f=\sqrt{u_{0}^{2}+v_{0}^{2}} \\
& \Phi=\operatorname{arctg}\left(\frac{v_{0}}{u_{0}}\right)
\end{aligned}
$$

and the spatial coordinates are expressed as:

$$
\begin{aligned}
& u_{0}=f \cos \Phi \\
& v_{0}=f \sin \Phi
\end{aligned}
$$

Using the previous equations, the complex sine wave is represented as:

$$
s(x, y)=e^{j(2 \pi f(x \cos \Phi+y \sin \Phi)+\varphi)}
$$

The 2D Gaussian function is defined as:

$$
w_{r}(x, y)=A e^{-\left(\frac{\left(x-x_{0}\right)^{2}}{2 \sigma_{x}^{2}}+\frac{\left(y-y_{0}\right)^{2}}{2 \sigma_{y}^{2}}\right)}
$$


where $\mathrm{A}$ is the amplitude, $\left(x_{0}, y_{0}\right)$ represents the center of the function, and $\sigma_{x}$ and $\sigma_{y}$ denotes the deviation of the Gaussian by each of the spatial coordinates.

Finally, after preliminary consideration, a function $g(x, y, f, \Phi, \sigma)$ represents the Gabor filter, where $\mathrm{f}$ is the spatial frequency, and $\Phi$ denotes the filter orientation [1-5]:

$$
g(x, y, f, \Phi, \sigma)=A e^{-\left(\frac{\left(x-x_{0}\right)^{2}}{2 \sigma_{x}^{2}}+\frac{\left(y-y_{0}\right)^{2}}{2 \sigma_{y}^{2}}\right)} e^{j(2 \pi f(x \cos \Phi+y \sin \Phi)+\varphi)}
$$

The deviation of the filter can be represented via the spatial frequency, $f$, i.e., the wavelength $\lambda$ measured in pixels, as [4]:

$$
\sigma_{x}=\lambda k_{x} \text { and } \sigma_{y}=\lambda k_{y}
$$

where $k_{x}$ and $k_{y}$ represent scaling factors for tuning the bandwidth of the filter. The fine tuning of these parameters is essential in order to adjust the Gabor filter and obtain appropriate filtering results [17-21].

Figure 1 shows a diagram of a Gabor filter with specified parameters in the spatial domain. This visualization in the spatial domain provides information on filter characteristics such as orientation and wavelength that can be easily modified by changing parameters of the 2D Gabor function to capture and emphasize different patterns in an image.

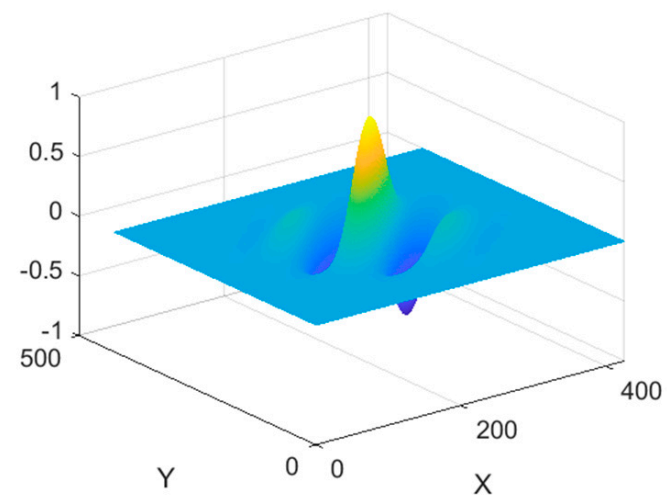

(a)

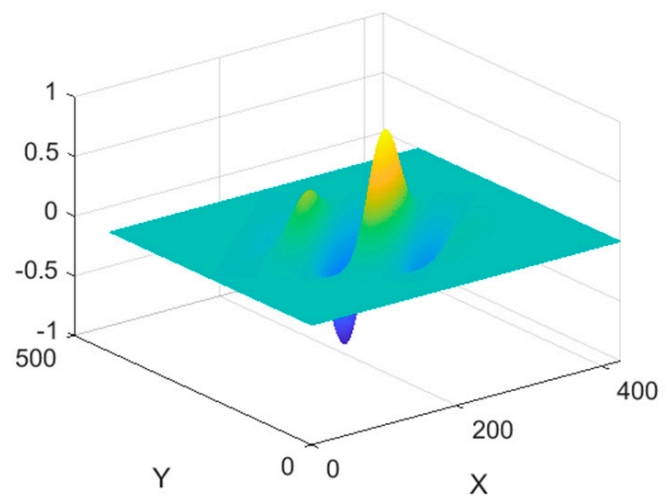

(b)

Figure 1. Example of the Gabor filter for $\lambda=15, \phi=45^{\circ}, k_{x}=k_{y}=0.5$; (a) even part; (b) odd part.

\subsection{Fuzzification Procedure-A Step by Step Guide}

As an improvement of the classical Gabor filter, Tadic et al. [17] introduced a new fuzzified Gabor filter. In their research, the goal was to develop a robust filtering approach for detection of license plates in complex, low-quality images. Using fuzzy logic [24,25], the orientation and wavelengths of the Gabor filter were fuzzified to improve the filter response [17-21]. It has been proven that these two parameters are major determinants in texture detection and extraction [17]. The experiments and the algorithm validation confirmed that Bell's function and the triangular membership function are the most efficient methods for selecting the filter parameters in the fuzzification process [17-21].

Hereinafter, the principle of the fuzzification of the Gabor filter parameters is explained as a guide for developing any fuzzy inference system [17-21]. In general, the fuzzification process can be summarized in five steps, elaborated in the following subsections [21].

\subsubsection{Fuzzification of the Input Parameters}

Fuzzification of input parameters: in this step, for each scalar input, the corresponding fuzzy value has to be found by mapping the given input data into the interval $[0,1]$ using the corresponding membership function for each rule. The membership functions map the input value to the degree of truth of a statement; thus, this step results in a representation of crisp quantities in a form that is applicable in the fuzzy logic [21]. In this work on 
the fuzzification of the Gabor filter parameters (orientation and wavelength), a Bell-type membership function has been selected as the input membership function for orientations and for the wavelengths:

$$
\mu_{i n}(\phi)=\left\{\begin{array}{cc}
S\left(\phi, \phi_{1}, \phi_{2}\right) & \phi<\phi_{2} \\
S\left(2 \phi_{2}-\phi, \phi_{1}, \phi_{2}\right) & \phi_{2} \leq \phi
\end{array}\right.
$$

where the interval of the possible orientation is $\phi \in\left[\phi_{1}, \phi_{2}\right]$, and $S$ denotes the S-shaped membership function [21]. The definition of the Bell-type function via S-shaped function is a common practice in literature [21]:

$$
S\left(\phi, \phi_{1}, \phi_{2}\right)=\left\{\begin{array}{cc}
0 & \phi<\phi_{1} \\
2\left(\frac{\phi-\phi_{1}}{\phi_{2}-\phi_{1}}\right)^{2} & \phi_{1} \leq \phi<\phi_{c} \\
1-2\left(\frac{\phi-\phi_{2}}{\phi_{2}-\phi_{1}}\right)^{2} & \phi_{c} \leq \phi<\phi_{2} \\
1 & \phi_{2} \leq \phi
\end{array}\right.
$$

The $\phi_{c}$ is called the crossover point of the S-shaped function [21]. This is the point where the S-shaped curve changes inflection. It is determined as [21]:

$$
\phi_{c}=\frac{\phi_{1}+\phi_{2}}{2}
$$

To improve the filtering, the interval of all possible orientations of the filter should be small, or a smaller number of adjacent orientations should be used to facilitate the localization of object of interest in the later stages. For these purposes, the triangular shape membership function is used [17-21] as the output membership function to reduce the input interval of the orientations and later, the wavelengths defined by the Bell-type membership function, and it is defined as [21]:

$$
\mu_{\text {out }}(\phi)=\left\{\begin{array}{cc}
0 & \phi<\phi_{i} \\
\left(\frac{\phi-\phi_{i}}{\phi_{\text {crisp }}-\phi_{i}}\right)^{2} & \phi_{i} \leq \phi<\phi_{\text {crisp }} \\
1-\left(\frac{\phi-\phi_{\text {crisp }}}{\phi_{f}-\phi_{\text {crisp }}}\right)^{2} & \phi_{\text {crisp }} \leq \phi<\phi_{f} \\
0 & \phi_{f} \leq \phi
\end{array}\right.
$$

where the $\phi_{i}$ and $\phi_{f}$ are the start-point and endpoint, respectively, of the reduced orientation interval defined by the output triangular shape membership function. The $\phi_{c r i s} p$ corresponds to the maximum of the triangular membership function, and it is determined as arithmetic mean of the $\phi_{i}$ and $\phi_{f}[21]$.

For the fuzzification of the wavelengths, the procedure implies some minor changes which arise primarily due to the nature of the variable. Generalized Bell functions were used to define two membership functions covering the input domain. In addition, two output segments and the triangular membership function were used to describe the output and achieve the smallest deviations from the central wavelength values.

It is worth noting that these functions fully describe the input and the output of the fuzzy system, and for this specific function selection they are presented in Figures 2 and 3.

\subsubsection{Fuzzy Logical Operations}

The second step in the process assumes performing any required fuzzy logical operations. In this step, it is necessary to combine the output data after fuzzification from all processed inputs using the corresponding membership functions (IF part of the rule, which describes the conditions) to get a single value using the operations max or min, depending on whether the parts are connected via logical addition (OR, Union) or multiplication (AND, Intesection), respectively [21]. The number of conditions (IF part of the rule) and the 
type of logical operator used for the connection may be different for each of the rules [21]. The max and min operations are defined as follows [21]:

$$
\begin{aligned}
& \mu_{U}(\phi)=\max \left[\mu_{A}(\phi), \mu_{B}(\phi)\right] \\
& \mu_{I}(\phi)=\min \left[\mu_{A}(\phi), \mu_{B}(\phi)\right]
\end{aligned}
$$

where $U$ denotes the union, $I$ denotes the intersection, $A$ and $B$ are arbitrary sets, and $\mu$ denotes the result of OR and AND operations. It should be noted that in the examples with only one condition in the IF part of the rule, there is no need to use AND or OR operations; this was the case in the process of Gabor filter fuzzification [21].

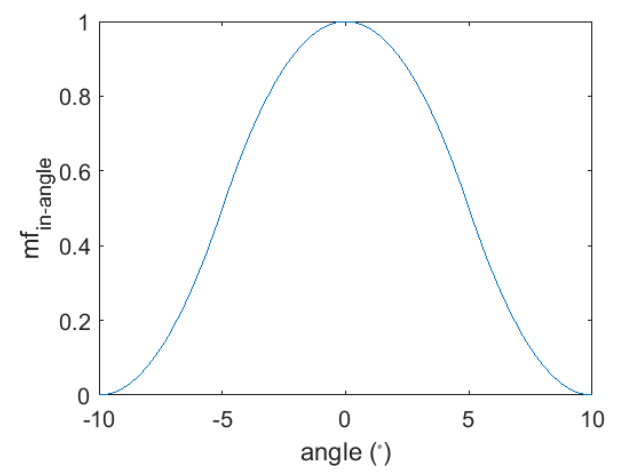

(a)

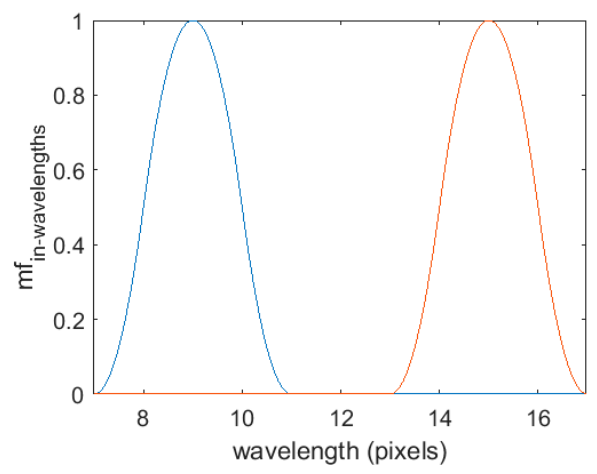

(b)

Figure 2. Example of the fuzzification process-input membership functions: (a) for the orientation, where the blue curve represents the interval covered by the membership function; (b) for the wavelength,where the blue and red curves represent the two intervals covered by two membership fuctions.

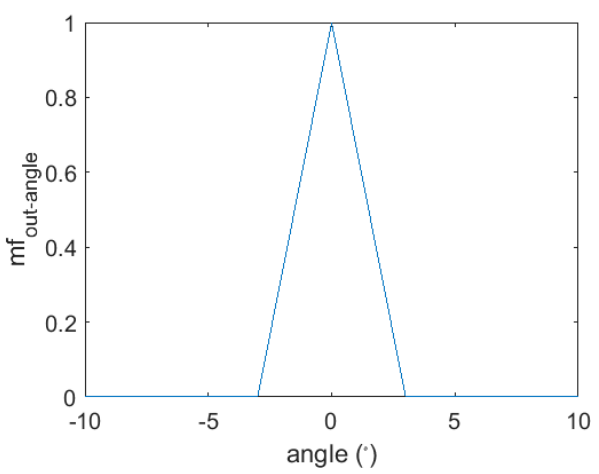

(a)

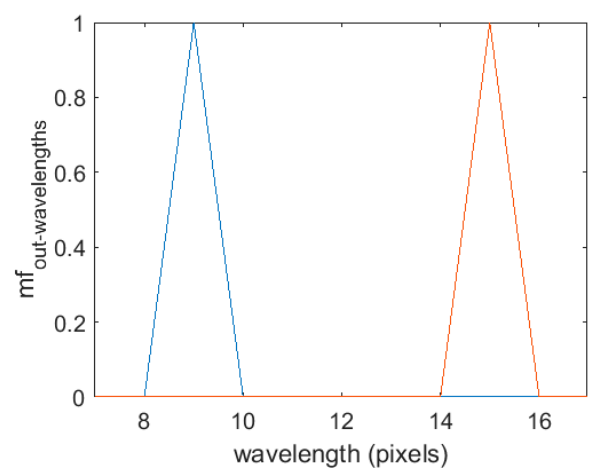

(b)

Figure 3. Example of the fuzzification process-output membership functions: (a) for the orientation, where the blue curve represents the interval covered by the membership function; (b) for the wavelength, where the blue and red curves represent the two intervals covered by two membership functions.

\subsubsection{Implication Step}

Application of the implication method: the single output after each condition (IF part of the rule) is used to provide the output of each rule. Logical multiplication (AND, min) or addition $(\mathrm{OR}, \max )$ operations are used for implication. This operation practically cuts off the corresponding output membership function in the values provided by the condition. Here, we get the output consequence of each rule which shows the extent of fulfilment of antecedent from the beginning of the rule [21].

According to steps 2 and 3, the If-THEN rules can be written as follows [21]:

$$
\text { IF }\left(\phi \text {, angle }_{\text {in }}\right) \text { THEN }\left(\phi, \text { angle }_{\text {out }}\right)
$$


for the orientations, and:

$$
\begin{aligned}
& \operatorname{IF}\left(\lambda \text {, wavelength } 1_{\text {in }}\right) \text { THEN }\left(\lambda, \text { wavelength }_{1 \text { out }}\right) \\
& \operatorname{IF}\left(\lambda \text {, wavelength } 2_{\text {in }}\right) \operatorname{THEN}\left(\lambda \text {, wavelength } 2_{\text {out }}\right)
\end{aligned}
$$

for the wavelengths, where $\phi$ and $\lambda$ represent orientation angles and wavelengths, respectively. The angle $e_{\text {in }}$ and angle $e_{\text {out }}$ represent the coresponding intervals of the input and output membership functions in fuzzification of orientations. The wavelength $1_{\text {in }}$ and wavelength $2_{\text {in }}$ and wavelength $1_{\text {out }}$ and wavelength $2_{\text {out }}$ represent the two intervals in input and output membership functions during the fuzzification process of wavelengths.

\subsubsection{Aggregation Step}

The fourth step of the process is the application of the aggregation method to the fuzzy sets obtained in step 3 [21]. In this step, the outputs of all rules that are now fuzzy sets are combined using the appropriate logical operations of addition (OR, max) or multiplication (AND, min). By combining the outputs of all the rules, a compact mathematical representation of the entire knowledge base is obtained, which basically represents the cross section of a surface [21]. In our research, the OR operation was used in an aggregation step, and it should be noted that the aggregation was performed similarly for both fuzzy systems, i.e., angles and wavelengths [17-19].

\subsubsection{Defuzzification Step}

The final step in creating a fuzzy system is the defuzzification of the final output fuzzy set. Defuzzification of the final fuzzy set is the opposite process of the fuzzification. In fact, this is a process that is supposed to convert the result of aggregation, which is basically a cross section of a surface, into a function, a signal that is understandable to the process. In this step, the fuzzy output (signal, function) is transformed into a clear (crisp), scalar form [21]. The output must have a single value, usually represented by a real number. This is necessary since all processes understand and accept only scalar values as inputs. The most often used method for defuzzification is the center of the gravity [21], and this defuzzification method is used for both fuzzy systems for the angles and wavelengths [17-19].

Using the described procedure, two fuzzy systems were obtained that described the orientation and the wavelength changes. The corresponding fuzzy inference systems (FIS) are shown Figure 4.

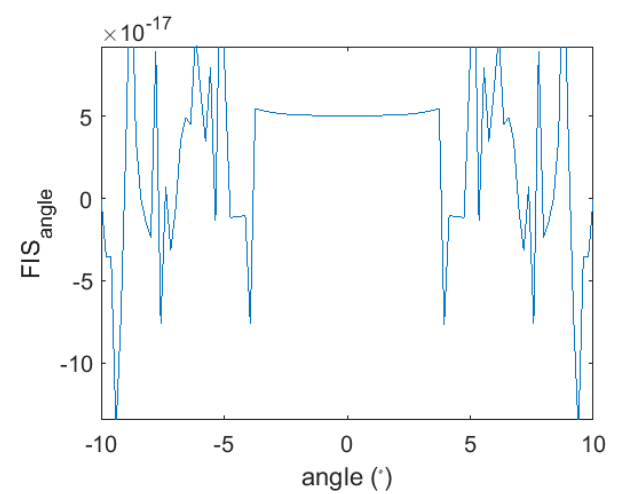

(a)

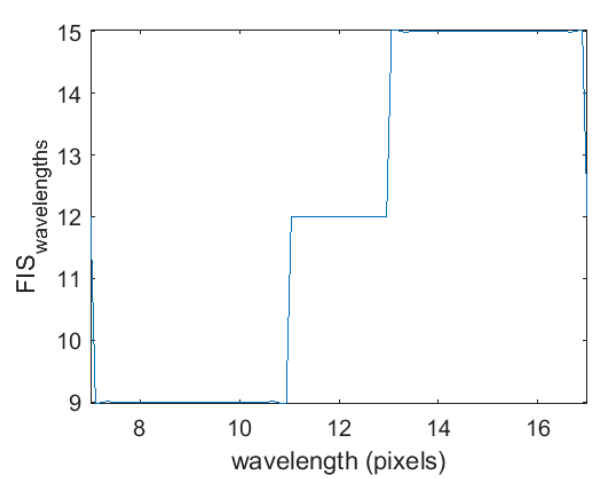

(b)

Figure 4. The developed fuzzy inference systems (FIS) with their fuzzy values: (a) for the orientation and (b) for the wavelength.

Once the fuzzification of the Gabor filter was completed, the new fuzzy Gabor filter was developed with fuzzified parameters. After filtering the input images with the fuzzified Gabor filter, the textures were detected and highlighted in the complex images. 
It is worth noting that the membership functions described above are not unique, and they can be adjusted to a specific problem and application. However, the principle of the fuzzification method is unchanged. This indicates the simplicity and the versatility of the application of the fuzzy logic in image processing, especially for object detection. Since the previous research is related to the detection of license plates in complex low-quality images [17-19], in Section 3, a series of examples will be presented to emphasize the fuzzy Gabor filter's capabilities in other applications as well [17-19]. In the end, it must be emphasized that the fuzzification was implemented in a Matlab environment $[17,21]$ using existing built-in functions from the scientific literature [21] to automatically generate the IFTHEN rules and create a fuzzy system providing input and output membership functions.

\section{Experiments and Results}

The application and performance of the fuzzy Gabor filter was illustrated in two examples of license plate detection tasks. The images used in this study were captured with a low-cost 3.1-megapixel Canon photo camera. Several images were taken with 2-megapixel mobile phone camera and with a commercial 10-megapixel Nikon photo camera.

\subsection{Comparison of the Gabor Filter and Fuzzy Gabor Filter-Qualitative Evaluation}

Figure 5 shows two images of cars captured during the day with a shadow effect [17]. The disadvantages of the low-quality camera image acquisition are noticeable in Figure 5, i.e., the shadow, noise, flash of a streetlamp, and various artefacts. These images were selected to demonstrate the performance and robustness of the fuzzified Gabor filter [17-19].

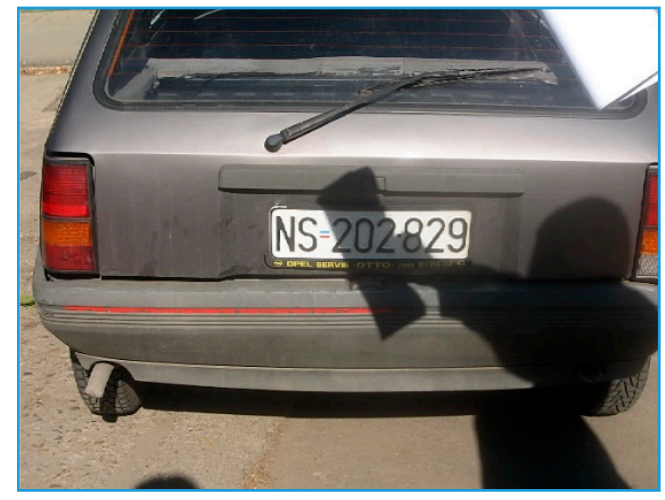

(a)

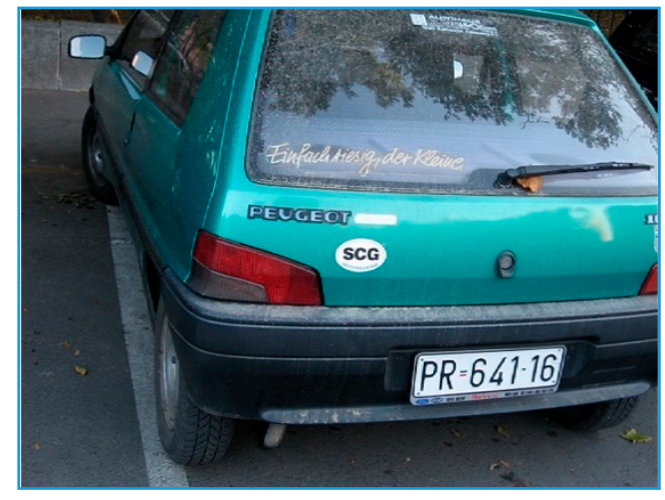

(b)

Figure 5. Example of the application of the fuzzy Gabor filter-original input images taken (a) in daylight with shadow and (b) in daylight.

In order to emphasize the improvement brought by the fuzzified Gabor filter, the input images were filtered using the classic Gabor filter and the fuzzy Gabor filter. Figure 6 shows the responses of the classic vertically oriented Gabor filter and of the fuzzy Gabor filter to the images presented in Figure 5. The classic Gabor filter without fuzzification had fixed parameters, orientation of $0^{\circ}$, wavelength of 15 pixels, and scale parameters of $k_{x}=k_{y}=0.5$. This classic Gabor filter was optimized for regular cases where the license plates and texts in the input images are under a clear horizontal angle without any slant or rotation. The results of the filtering with the classic Gabor filter (Figure 6a,b) highlighted the license plate area in both images.

Figure $6 c$,d show the results obtained using the fuzzified Gabor filter. Because the membership functions were chosen to emphasize the content of the license plate, it can be observed that the strongest responses were in the areas of the plates, and these results contained more detected textures compared to the output of the classic Gabor filter. Specially, the left parts of the license plates were highlighted with more detected textures. The filter adaptability to the application of interest was the goal of the fuzzification process. 
In addition, the robustness of the filter is obvious from these examples because the input images contained high noise and artefacts.

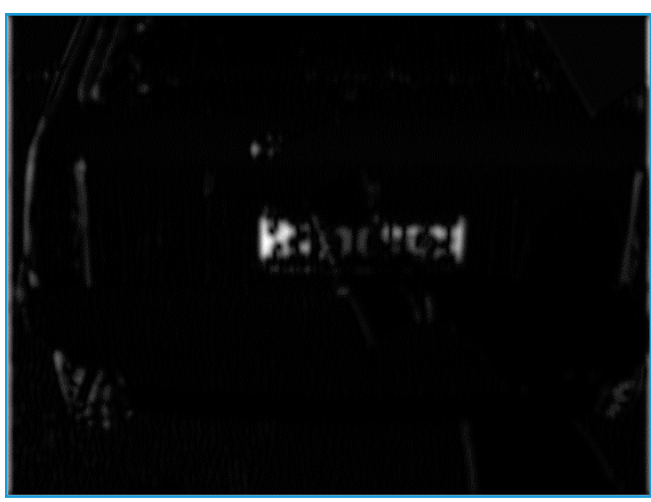

(a)

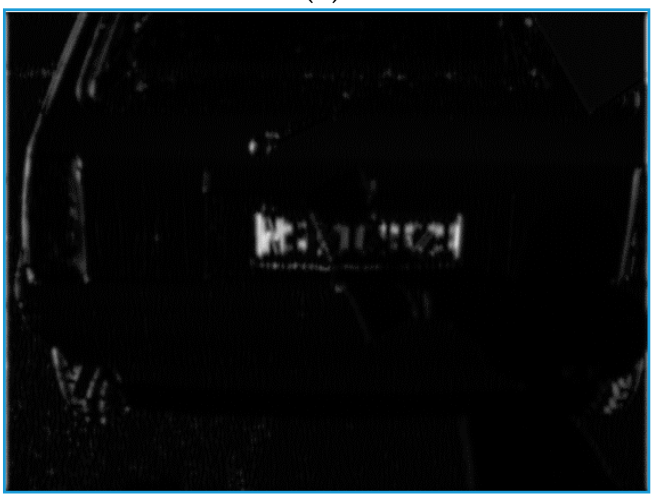

(c)

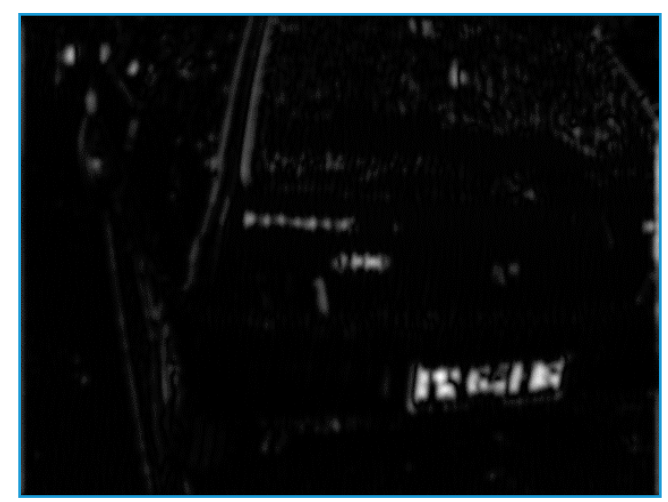

(b)

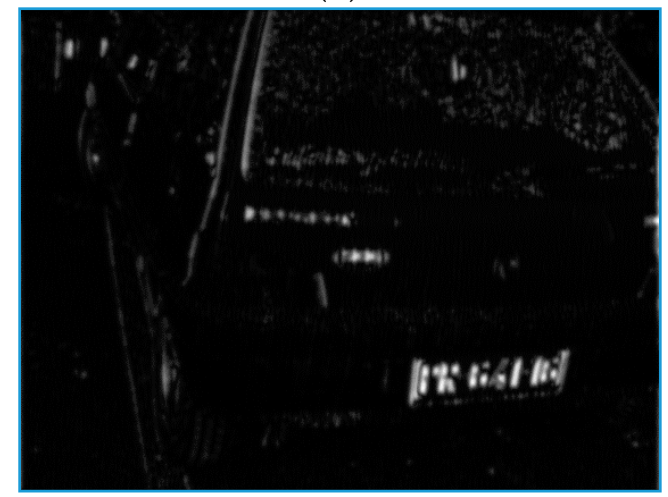

(d)

Figure 6. Response of the input image to the classic Gabor filter: (a) daylight image with shadow and (b) daylight image; response of the input image to the fuzzy Gabor filter: (c) daylight image with shadow and (d) daylight image.

Figure 7 presents the 3D projections of the corresponding responses from Figure 6 . In Figure $7 \mathrm{a}-\mathrm{d}$, the license plate areas were clearly distinguished. In Figure $7 \mathrm{~b}, \mathrm{~d}$, it can be noted that the license plate areas were clearly elevated from the image background, while in Figure $7 \mathrm{a}-\mathrm{c}$, the license plate areas were well elevated too, but the effects of the shadow could be noticed in the projection. Also, it should be emphasized that the maximal values of the license plate areas in Figure $7 \mathrm{c}, \mathrm{d}$ were higher and more equal in the examples related to the fuzzified Gabor filter. This was the intention during the deployment of the fuzzy-based algorithm, i.e., to reliably detect the textures in low-quality images using simple operations [17-19].

Finally, using the benefits of the response to the fuzzy Gabor filter from Figure 6, the license plates with the contained text were detected and extracted using a sequence of image processing operations, such as Sobel edge detection, threshold application, a sequence of morphological operations, connected-component analysis for frame detection, license plate and text marking, and finally, plate cropping (Figure 8c,d) [17]. Figure 8a,b show the extracted license plates after the filtering with the classical Gabor filter. In Figure 8a, the left part of the license plate was slightly cropped, but in Figure $8 b$, the license plate was more severely deficient. The results of the fuzzified approach (Figure $8 c, d$ ) indicate the improvements on both examples.

Several factors signoficantly influenced plate detection performance, such as the slant, skew, and the distortion of the vehicles position in the image [17-19]. These factors influenced the detection of the text in the image as well as any textures of interest [17-19]. The automatic slant detection and correction of objects of interest is a very common and difficult problem in object recognition. 


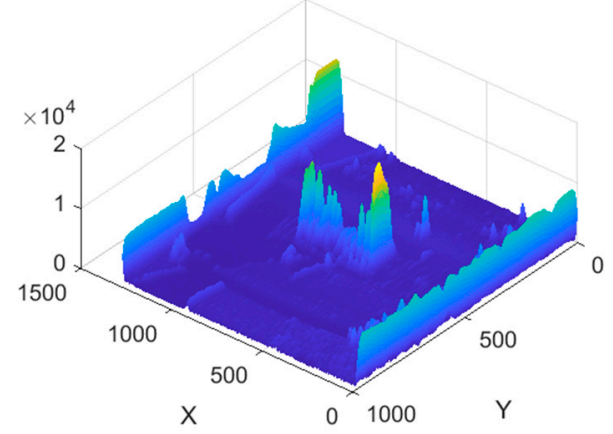

(a)

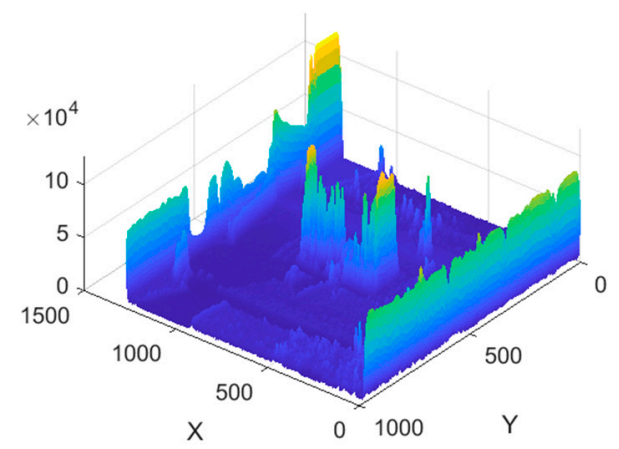

(c)

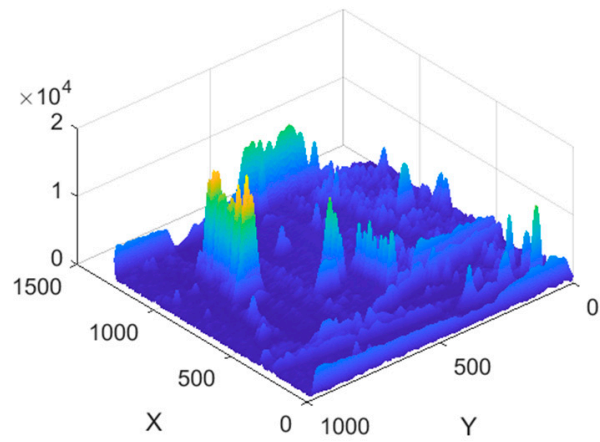

(b)

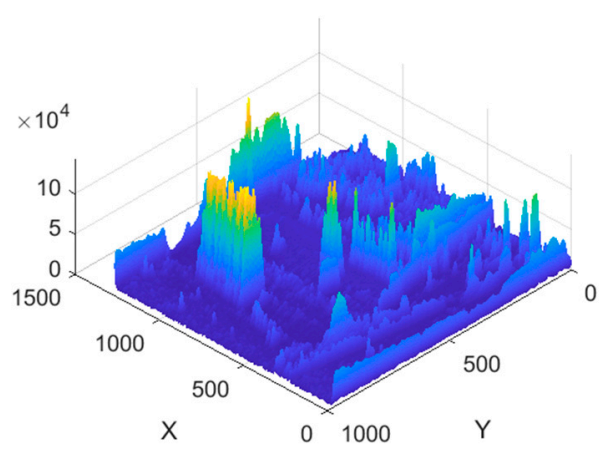

(d)

Figure 7. Projection of the response to the classic Gabor filter: (a) daylight image with shadow and (b) daylight image; projection of the response to the fuzzy Gabor filter: (c) daylight image with shadow and (d) daylight image.

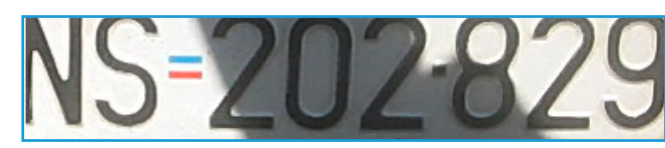

(a)

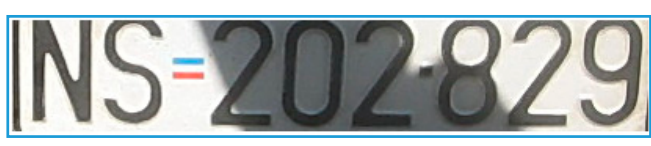

(c)

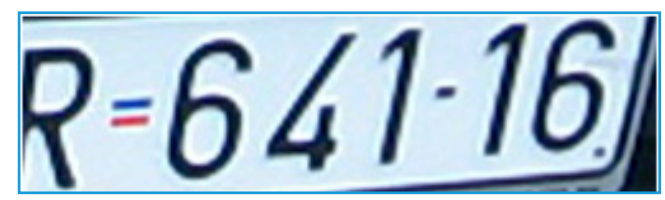

(b)

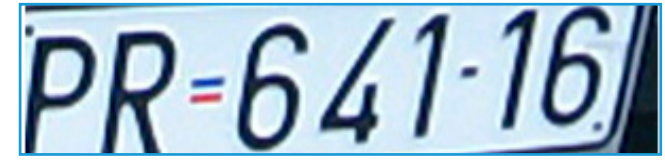

(d)

Figure 8. The algorithm outputs - the extracted license plate with text: (a) filtered with classic Gabor filter: daylight image with shadow, (b) filtered with classic Gabor filter: daylight image, (c) filtered with fuzzy Gabor filter: daylight image with shadow, and (d) filtered with fuzzy Gabor filter: daylight image.

To explore the effectiveness of a fuzzified Gabor filter, a series of experiments is presented in Figures 9 and 10.

Figure 9 shows the results of license plate detection from images captured under various lighting conditions from various positions at a pronounced oblique angle in the first three examples. The fourth example contains an image with a high degree of glare which made the content of the license plate almost unvisible. As it can be seen, all the presented images contained significant shortcomings, glare, skew, noise, and artefacts. In the first example, the image was captured at night with an old mobile phone camera. A street lamp about $10 \mathrm{~m}$ away provided low light on the left side of the vehicle. Also, the car and its license plate were at a pronounced oblique angle that was obviously greater than the assumed $10^{\circ}$ in which the interval of the input membership function was defined [17-19]. 
Despite a number of shortcomings in the input image, the fuzzifed Gabor filter highlighted the desired textures belonging to the license plate area, and the license plate was successfully detected and isolated. Only a very small part of the plate was cut off from the left side. The second example in Figure 9 shows a car photo captured at daylight under good lighting conditions, but the car and the license plate were again at skew angle. Since this rotation angle was smaller than in the previous example, the fuzzy Gabor filter detected the license plate without damage. The third example displays a rotated image of the license plate in three-dimensions (3D). The rotation in the $X-Y$ plane was about $15^{\circ}$ or even more. The goal of this example was to show the filtering effects in the case where the image of the vehicle was markedly rotated in relation to the normal position [17-19]. As it can be noticed, the fuzzified filter emphasized the license plate area with its textures in a great manner. Based on the detected textures, the license plate was extracted again with a slight cut-off of the number on the right side of the plate. Despite this cut-off, the content of the license plate was fully recognizable. It should be noted that these examples do not present a regular case, but rather demonstrate different limit cases in which the algorithm could still provide acceptable results.
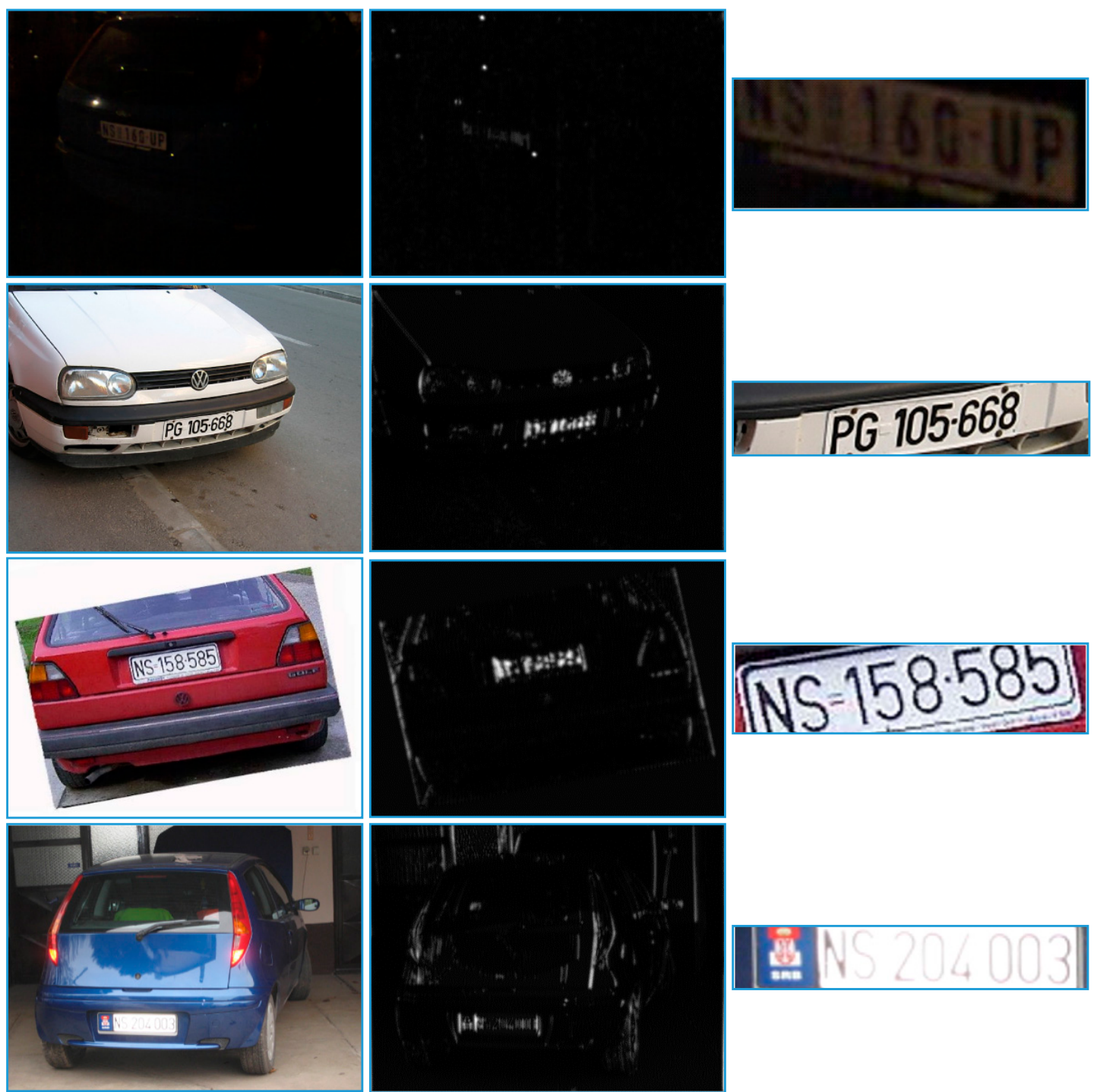

(a)

(b)

(c)

Figure 9. The results of the algorithm with fuzzified Gabor filter: (a) original input images, (b) results of the filtering with fuzzy Gabor filter, and (c) detected license plates. 

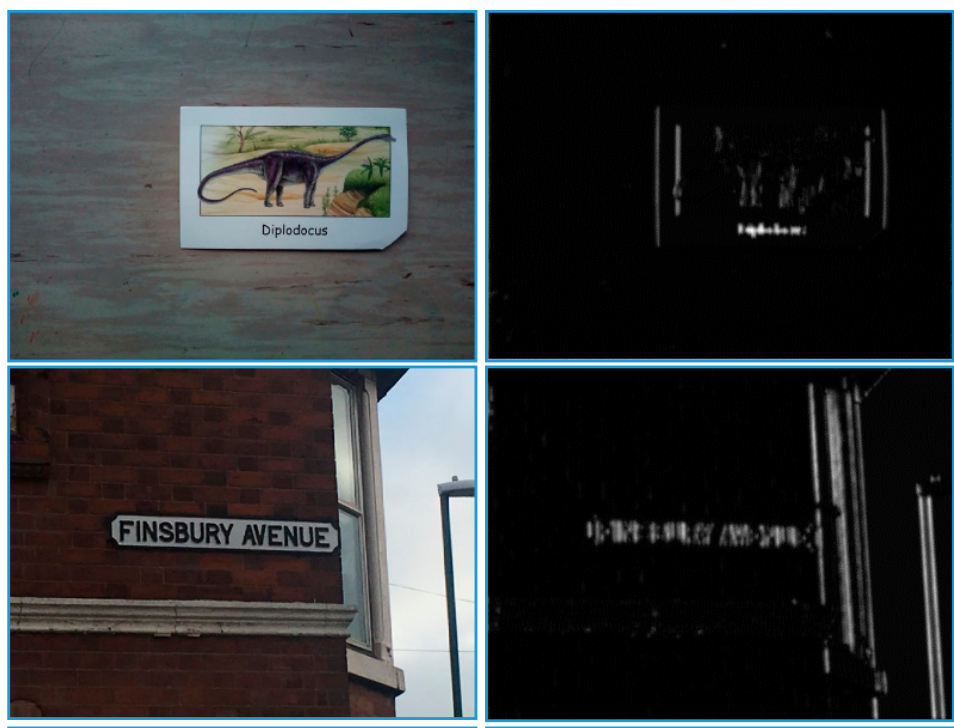

\section{Diplodocus}
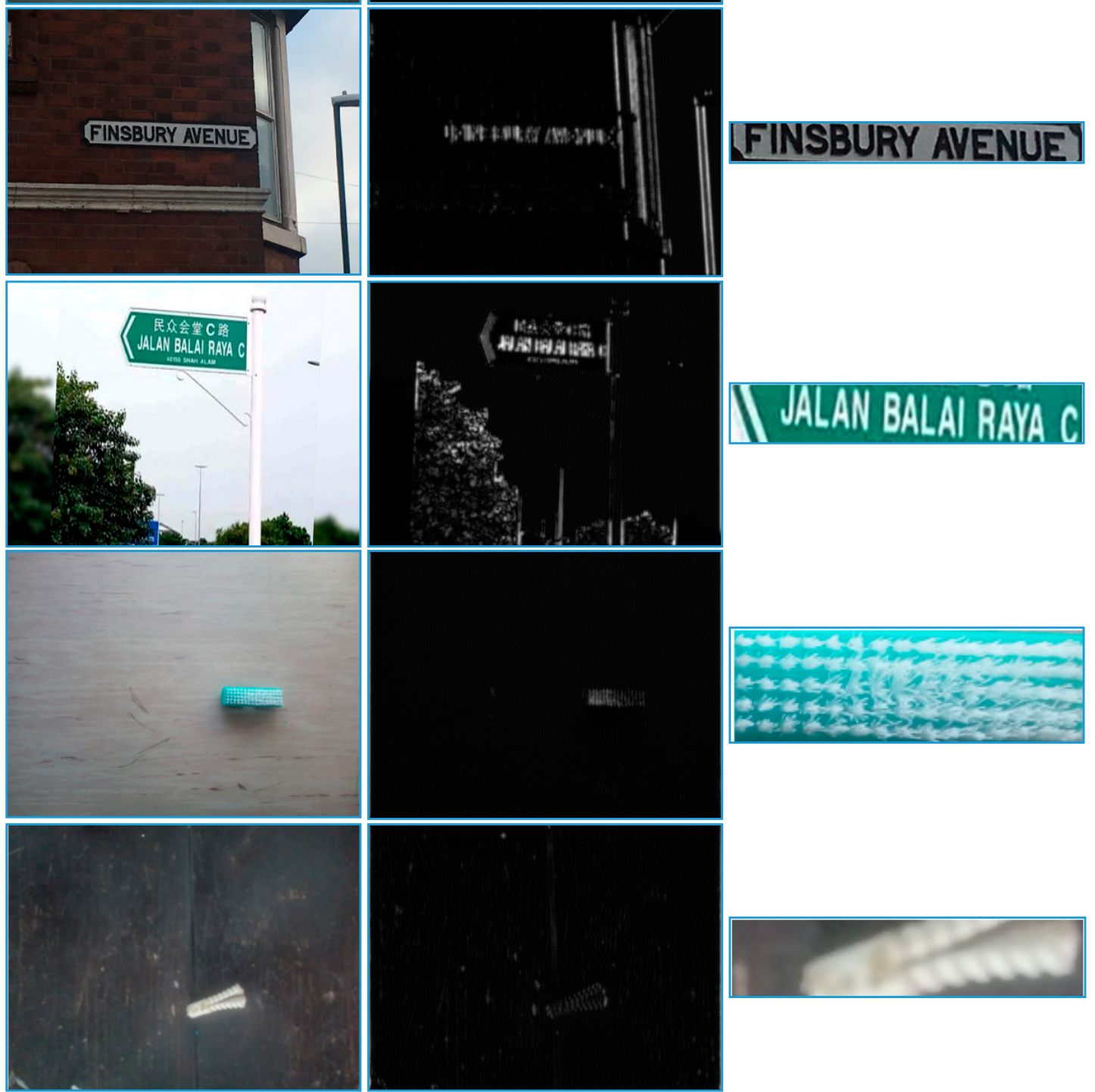

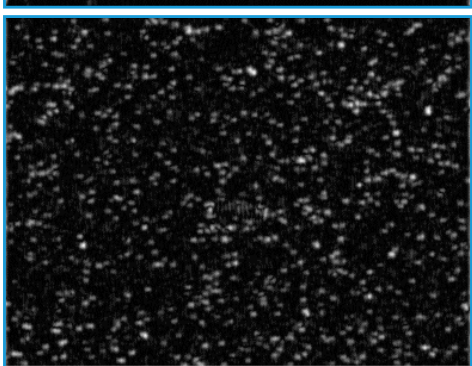

(b)

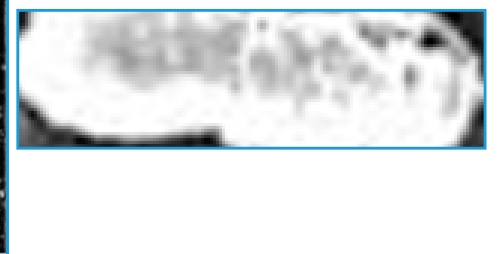

(c)

Figure 10. The results of the algorithm with fuzzified Gabor filter: (a) original input images, (b) results of the filtering with fuzzy Gabor filter, and (c) detected text areas and detected objects with appropriate textures. 
Finally, the example in the last row of Figure 9a shows a car captured under pronounced sun rays, which resulted in an image with light reflection and glare. The content of the license plate was not visible properly. Despite the glare, the fuzzified Gabor filter detected the desired textures in the plate area, and the license plate was extracted. The presented results prove the robustness of the fuzzy Gabor filter on various image recording conditions. These examples further confirm the results obtained in the previous research [17-19].

In this work the experiments were further extended on text detection in various signs and inscriptions. Since the text area in these examples was similar to the text in license plates, the fuzzified Gabor filter and the proposed extraction algorithm could be used for the detection of text in images. Figure 10 presents several examples of images (column a), with fuzzified Gabor filter output (column b) and extracted text components. The first example in Figure 10 shows a children's dinosaur map with an inscription. The image was captured under poor lighting conditions with a modest quality photo camera. After the filtering, the fuzzified filter successfuly extracted the textures in the inscription area, and the text was detected and extracted without any imperfection. The second example diplays a part of a building with a street sign. The street sign and all the other horizontal lines in the image were slightly rotated. The fuzzy Gabor filter perfectly highlighted the street name area, facilitating the text detection without any significant imperfections. The third example presents a road sign under a skew angle. The image was captured at daylight; it contained blured parts from both sides, and the origin of the photo is unknown. The fuzzified Gabor filter detected the textures in text area to a large extent and as a final result, the desired text area was extracted with a very small cut off in the lower part of the letter " $\mathrm{C}$ ".

The following three examples in Figure 10 show various textures, presented as examples of texture detection applications. Since the Gabor filter is a robust texture detector, the goal of the presented expiriments was to demonstrate performance on the examples related to change in texture. The fourth image in Figure 10 shows a nailbrush from its brush-side. The image was captured with a poor quality camera at daylight and with a shadow from left. The brush was slightly rotated, and the background had variegated textures. As it can be seen, the fuzzy Gabor filter detected the textures in the brush area very well, and the textures belonging to the brush were clearly visible. Based on these detected textures, the brush was extracted from the background. The fifth example in Figure 10 displays a plastic dowel, captured at poor lighting conditions and at an excessive slant compared to the horizontal position, which was initially set as a in the license plate detection problem. In the correponding column b image, it is noticable that the fuzzified Gabor filter highlighted the textures related to the dowel area, which were distinguishable relative to the background. The dowel was extracted (the corresponding column c image) with small imperfections on the left lower and right upper sides due to an excessive rotation of the dowel in the original input image. This was an expected result since the percent interval of the angles taken into account were between $-10^{\circ}$ and $10^{\circ}$. The final example in Figure 10 shows an Esherichia bacteria in variegated background. The image is of unknown origin; this is probably a microscopic image or a modeled image. The fuzzy filter detected the textures of the desired shape, especially those vertically oriented, yet due to the variagated background, the textures related to the bacteria area were not clearly visible. The bacteria was extracted from the background based on the poorly detected textures.

As illustrated in the examples in Figures 9 and 10, the fuzzified Gabor filter has shown its robustness with respect to the ambient conditions during image aquisition, and detected the textures of interest in a very high quality manner. The algorithm initally developed and tuned for license plate detection [17-19] has been successfully applied to the detection of text and incriptions and even some shapes that contained textures similar to license plates. All these results support the usefulness of the fuzzy Gabor filter in texture detection and open possibilities for the utilization of this fuzzy filter in other applications with certain changes in the parameter fuzzification. 


\subsection{Comparison of Gabor Filter and Fuzzy Gabor Filter-Quantitative Evaluation}

In the end, the results of the quantitive comparions between the fuzzy Gabor filter and the classic Gabor filter will be presented. Since the research was initially related to license plate detection, both algorithms were validated on a dataset of 718 vehicle images captured with a classical modest-quality digital camera (appl-dsp.com/wpcontent/uploads/2019/06/test\%20database2.zip (accessed on 25 March 2021.)) [17]. The images were captured during a day and night with poor lighting conditions. The presumption was that the skewness of the license plates was approximately in interval of $(-1010)$ degrees. The supposed distance between the vehicles and camera was approximately between 2-10 $\mathrm{m}$. These image aquisition conditions are important, since the fuzzification and the algorithm were developed for certain application requirements. Following the fuzzification guidelines, the filter can be modified to meet different application demands, and a novel, more suitable test dataset can be produced. These approaches tailored for specific applications would yield an improved performance over universal, potentially suboptimal solutions.

The percentage of correctly localized license plates is a usually used measure for the evaluation of procedures for license plate detection. A correct license plate localization means the detection of a plate that facilitates full visibility of its contents, sufficient for a perfect license number recognition [17]. Table 1 displays a performance summary of the two filtering approaches. The performance improvement, as measured by the percentage of correct detection of a license plates from a complex images, has been noted in a fuzzified Gabor filtering approach.

Table 1. Summary of the detection results for the classic Gabor filter and fuzzy Gabor filter.

\begin{tabular}{cccc}
\hline Type of Gabor Filter & Number of Images & Localized License Plates & Error (Miss Rate) \\
\hline Classic Gabor Filter & 718 & $86.6 \%$ & $13.4 \%$ \\
\hline Fuzzy Gabor Filter & 718 & $97.9 \%$ & $2.1 \%$ \\
\hline
\end{tabular}

The performance of the two presented algorithms was mainly similar to the performance of other license plate detection algorithms. The main advantage of the fuzzy Gabor filter based procedure is its simplicity and the high degree of robustness in the case of very poor quality input images, corupted with noise and artefacts.

The fuzzified Gabor filter used in this note yielded a markedly better performance as compared to the classic Gabor filter. A credible explanation is an improved robustness to the image capturing conditions enabled by a fuzzy logic. Hence, in applications where some parameters are imprecisely or incorrectly set (such as the input image for detection of license plates or text, where the precise angle of capturing, brightness, etc. are not known in advance), the use of the fuzzy reasoning is a good solution since the fuzzy system successfully uses human reasoning in solving the problem [17-21]. In the evaluation of a classic Gabor filter performance on the open dataset, more than $70 \%$ of the errors in localization were due to cut-off of the licence plate. The main reason for this underperformance was a fixed vertical orientation and a fixed wavelength. These rigid parameters are not favourable when there is an excessive slant, a pronounced influence of glare due to light sources, or the license plate is too large or too small due to the distance from which the vehicle was taken. This means that the capturing distance was outside the assumed distance interval. Hence, the errors were expected in certain examples, but the goal of the experiment was to test the limit performance of the fuzzified Gabor filter and compare it with the classic Gabor filter with fixed parameters. The obtained results and limitations will serve as guidelines in future research.

\section{Conclusions}

This note presents the advantages of the application of the fuzzy optimization principle to Gabor filter parameters. The fuzzification was conducted to adjust the filter parameters 
for filtering of poor-quality input images. The Gabor filter and the process of applying the fuzzy logic was summarized in brief. The advantages of the parameter fuzzification over the standard fixed Gabor filter parameters were confirmed by license plate detection results on the dataset of 718 images, and on a series of example images with text and different textures. These encouraging results will focus our future research on the fuzzification of other image processing filters.

Author Contributions: V.T. conceived and performed the experiments. A.O., Z.V. and A.T. checked the test results and suggested the corrections. T.L.-T., Z.T. and P.O. supervised the research and contributed to the organization of the article. V.T. and T.L.-T. drafted and revised the manuscript, and all authors approved the final version of the manuscript. All authors have read and agreed to the published version of the manuscript.

Funding: This research was funded by projects EFOP-3.6.3-VEKOP-16-2017-00009, EFOP-3.6.1-162016-00004, 2020-4.1.1-TKP2020, and 2020-1.1.2-PIACI-KFI-2020-00173, co-financed by the European Union and by the Ministry of Education, Science, and Technological Development of the Republic of Serbia through the project no. 451-03-68/2020-14/200156: “Innovative scientific and artistic research from the FTS (activity) domain".

Institutional Review Board Statement: Not applicable.

Informed Consent Statement: Not applicable.

Acknowledgments: The authors would like to thank the editors and the anonymous reviewers for their valuable comments that significantly improved the quality of this paper.

Conflicts of Interest: The authors declare no conflict of interest.

\section{References}

1. Daugman, J.G. Uncertainty relation for resolution in space, spatial frequency and orientation optimized by two-dimension visual cortical filters. J. Opt. Soc. Am. 1985, 2, 1160-1169. [CrossRef]

2. Tang, Q.; Sang, N.; Zhang, T. Extraction of salient contours from cluttered scenes. Pattern Recognit. 2007, 40, 3100-3109. [CrossRef]

3. Mehrotra, R.; Namuduri, K.; Ranganathan, N. Gabor filter-based edge detection. Pattern Recognit. 1992, 25, 1479-1494. [CrossRef]

4. Movellan, J.R. Tutorial on Gabor Filters; Kolmogorov Project, Machine Perception Laboratory: San Diego, CA, USA, 2006.

5. Liu, S.-L.; Niu, Z.-D.; Sun, G.; Chen, Z.-P. Gabor filter-based edge detection: A note. Optik 2014, 125, 4120-4123. [CrossRef]

6. Allagwail, S.; Gedik, O.S.; Rahebi, J. Face Recognition with Symmetrical Face Training Samples Based on Local Binary Patterns and the Gabor Filter. Symmetry 2019, 11, 157. [CrossRef]

7. Yu, M.; Zhang, Y.; Li, Y.; Lin, Z.-L.; Li, J.; Wang, C. Saliency Guided Visual Tracking via Correlation Filter with Log-Gabor Filter. IEEE Access 2020, 8, 158184-158196. [CrossRef]

8. Zhang, Y.; Li, W.; Zhang, L.; Ning, X.; Sun, L.; Lu, Y. Adaptive Learning Gabor Filter for Finger-Vein Recognition. IEEE Access 2019, 7, 159821-159830. [CrossRef]

9. Wu, J.; Wei, P.; Yuan, X.; Shu, Z.; Chiang, Y.-Y.; Fu, Z.; Deng, M. A New Gabor Filter-Based Method for Automatic Recognition of Hatched Residential Areas. IEEE Access 2019, 7, 40649-40662. [CrossRef]

10. Xiao, J.; Duan, X.; Qi, X.; Wang, J. Direction navigability analysis of geomagnetic field based on Gabor filter. J. Syst. Eng. Electron. 2018, 29, 378-385. [CrossRef]

11. Low, C.-Y.; Teoh, A.B.-J.; Ng, C.-J. Multi-Fold Gabor, PCA, and ICA Filter Convolution Descriptor for Face Recognition. IEEE Trans. Circuits Syst. Video Technol. 2017, 29, 115-129. [CrossRef]

12. Capizzi, G.; Sciuto, G.L. A Novel 2-D FIR Filter Design Methodology Based on a Gaussian-Based Approximation. IEEE Signal Process. Lett. 2019, 26, 362-366. [CrossRef]

13. Luan, S.; Chen, C.; Zhang, B.; Han, J.; Liu, J. Gabor Convolutional Networks. IEEE Trans. Image Process. 2018, $27,4357-4366$. [CrossRef]

14. Bai, X.; Ye, L.; Zhu, J.; Zhu, L.; Komura, T. Skeleton Filter: A Self-Symmetric Filter for Skeletonization in Noisy Text Images. IEEE Trans. Image Process. 2019, 29, 1815-1826. [CrossRef]

15. Medina, R.; Llamas, J.; Gómez-García-Bermejo, J.; Zalama, E.; Segarra, M.J. Crack Detection in Concrete Tunnels Using a Gabor Filter Invariant to Rotation. Sensors 2017, 17, 1670. [CrossRef]

16. Meng, X.; Yin, Y.; Yang, G.; Xi, X. Retinal Identification Based on an Improved Circular Gabor Filter and Scale Invariant Feature Transform. Sensors 2013, 13, 9248-9266. [CrossRef]

17. Tadic, V.; Popovic, M.; Odry, P. Fuzzified Gabor filter for license plate detection. Eng. Appl. Artif. Intell. 2016, 48, 40-58. [CrossRef]

18. Tadic, V.; Odry, A.; Toth, A.; Vizvari, Z.; Odry, P. Fuzzified Circular Gabor Filter for Circular and Near-Circular Object Detection. IEEE Access 2020, 8, 96706-96713. [CrossRef] 
19. Tadic, V.; University of Dunaújváros; Kiraly, Z.; Odry, P.; Trpovski, Z.; Loncar-Turukalo, T. University of Novi Sad Comparison of Gabor Filter Bank and Fuzzified Gabor Filter for License Plate Detection. Acta Polytech. Hung. 2020, 17, 61-81. [CrossRef]

20. Zadeh, L.A. Fuzzy Sets. Inf. Control 1965, 8, 338-353. [CrossRef]

21. Gonzales, R.C.; Woods, R.E.; Steven, L.; Eddins, S.L. Digital Image Processing Using MATLAB, 2nd ed.; Gatesmark, LLC: Knoxville, TN, USA, 2009.

22. Saha, S.; Ghosh, M.; Ghosh, S.; Sen, S.; Singh, P.K.; Geem, Z.W.; Sarkar, R. Feature Selection for Facial Emotion Recognition Using Cosine Similarity-Based Harmony Search Algorithm. Appl. Sci. 2020, 10, 2816. [CrossRef]

23. Wang, M.; Gao, L.; Huang, X.; Jiang, Y.; Gao, X. A Texture Classification Approach Based on the Integrated Optimization for Parameters and Features of Gabor Filter via Hybrid Ant Lion Optimizer. Appl. Sci. 2019, 9, 2173. [CrossRef]

24. Zhu, E.; Yin, J.; Zhang, G.; Hu, C. A Gabor Filter Based Fingerprint Enhancement Scheme Using Average Frequency. Int. J. Pattern Recognit. Artif. Intell. 2006, 20, 417-429. [CrossRef]

25. Ngo, T.H.; Seo, M.; Matsushiro, N.; Xiong, W.; Chen, Y.-W. Quantitative analysis of facial paralysis based on limited-orientation modified circular Gabor filters. In Proceedings of the 2016 23rd International Conference on Pattern Recognition (ICPR), Cancún, México, 4-8 December 2016.

26. Han, J.; Ma, K.-K. Rotation-invariant and scale-invariant Gabor features for texture image retrieval. Image Vis. Comput. 2007, 25, 1474-1481. [CrossRef]

27. Manthalkar, R.; Biswas, P.; Chatterji, B. Rotation invariant texture classification using even symmetric Gabor filters. Pattern Recognit. Lett. 2003, 24, 2061-2068. [CrossRef]

28. Wicaksono, Y.; Wahono, R.S.; Suhartono, V. Color and Texture Feature Extraction Using Gabor Filter-Local Binary Patterns for Image Segmentation with Fuzzy C-Means. J. Intell. Syst. 2015, 1, 15-23.

29. Ghandehari, A.; Safabakhsh, R. Palmprint Verification Using Circular Gabor Filter. In International Conference on Biometrics; Tistarelli, M., Nixon, M.S., Eds.; ICB 2009, LNCS 5558; Springer: Berlin/Heidelberg, Germany, 2009; pp. 675-684.

30. Kassis, M.; El-Sana, J. Scribble Based Interactive Page Layout Segmentation Using Gabor Filter. In Proceedings of the 15th International Conference on Frontiers in Handwriting Recognition, Shenzhen, China, 23-26 October 2016. [CrossRef]

31. Sevastjanov, P.; Dymova, L.; Bartosiewicz, P. A new approach to normalization of interval and fuzzy weights. Fuzzy Sets Syst. 2012, 198, 34-45. [CrossRef]

32. Gong, K.; Chen, C. A Programming-Based Algorithm for Probabilistic Uncertain Linguistic Intuitionistic Fuzzy Group DecisionMaking. Symmetry 2019, 11, 234. [CrossRef]

33. Raghunandan, K.S.; Shivakumara, P.; Jalab, H.A.; Ibrahim, R.W.; Kumar, G.H.; Pal, U.; Lu, T. Riesz Fractional Based Model for Enhancing License Plate Detection and Recognition. IEEE Trans. Circuits Syst. Video Technol. 2017, 28, 2276-2288. [CrossRef]

34. Rafique, M.A.; Pedrycz, W.; Jeon, M. Vehicle license plate detection using region-based convolutional neural networks. Soft Comput. 2017, 22, 6429-6440. [CrossRef]

35. Corneto, G.L.; Da Silva, F.A.; Pereira, D.R.; De Almeida, L.L.; Artero, A.O.; Papa, J.P.; De Albuquerque, V.H.C.; Sapia, H.M. A New Method for Automatic Vehicle License Plate Detection. IEEE Lat. Am. Trans. 2017, 15, 75-80. [CrossRef] 